\title{
2 An Evaluation of the Response Modulation Hypothesis in Relation to Attention-Deficit/Hyperactivity Disorder
}

4 Richard F. Farmer $\cdot$ Julia J. Rucklidge

R. F. Farmer $(\bowtie)$

Oregon Research Institute,

1715 Franklin Blvd., Eugene, Oregon 97403

e-mail: rfarmer@ori.org

\section{J. J. Rucklidge}

University of Canterbury and Youth Specialty Service,

Canterbury District Health Board,

Christchurch, New Zealand central emphasis on deficits in behavioral inhibition (e.g., Barkley, 1997; Oosterlaan \& Sergeant, 1996; Quay, 1997). Behavioral inhibition is a relatively broad concept and, as outlined in Barkley (1997), is manifest in at least three forms. One form is evident in the delay of prepotent (reinforced) responses. Without inhibition and delay, ongoing behavior would be largely influenced by immediate reinforcing events and have an automatic or stimulus-response quality, whereby the onset of a stimulus cue associated with reward would result in an immediate response instrumental in producing rewards signaled by the cue. In the absence of inhibition and delay, the opportunity to evaluate behavior in relation to longerterm or distal outcomes becomes compromised; that is, the ability to engage in goal-directed behavior becomes impaired. Inhibition and delay of ongoing behavior allows for the opportunity to notice whether behavioral outcomes are consistent with distal behavioral objectives, and whether the immediate consequences that behavior produces contrast or are consistent with these longer-term goals (Barkley, 1997).

Another form of behavioral inhibition is the interruption of ongoing behavior (Barkley, 1997). The interruption or delay of ongoing behavior allows for the possibility of adjusting behavior that is no longer effective or adaptive when environmental contingencies shift. Perseverative behavior that continues without interruption or influence by changing environmental events is often rigid in form and non-adaptive in function. Finally, interference control refers to the process of protecting goal-directed behavior from interference or disruption from competing events. Examples of this would include resistance from distraction when potential distracters are not relevant for ongoing goal-directed behavior, or the inhibition of motor actions that are inappropriate to a task or goal (Barkley, 1997).

A considerable body of research has demonstrated pervasive deficits in behavioral inhibition among youth with 
ADHD. For example, research investigations that have employed the "stop task," a common experimental paradigm where participants quickly inhibit prepotent responses to a "go" signal when signaled to do so by a "stop" (or "no-go") signal, repeatedly demonstrate that youth with ADHD have difficulties with response inhibition compared to other groups (e.g., Rucklidge \& Tannock, 2002). However, these studies also demonstrate that deficits in response inhibition may not be a unique and distinguishing feature of ADHD, as these deficits are also evident in children with conduct disorder but not anxiety disorders (Oosterlaan, Logan, \& Sergeant, 1998). Deficits in response inhibition, therefore, might constitute a characteristic feature common to individuals with externalizing disorders and associated behavior patterns.

Emotional/motivational theories of ADHD that emphasize impairments in inhibition processes are exemplified in the work of Quay $(1988,1997)$, who has firmly embedded his theory of ADHD within Gray's neuropsychological model. Briefly, Gray $(1970,1987)$ has delineated a conceptual brain system, termed the behavioral inhibition system (BIS), that he hypothesized (a) inhibits behavior in situations where cues associated with punishment are present, (b) increases arousal to energize subsequent behavior, and (c) increases attentional resources to initially threatening novel stimuli. High levels of anxiety as well as heightened sensitivity or responsiveness to the effects of punishment or frustrative non-reward (i.e., the non-occurrence of an expected reward) are associated with the activation of the BIS. A weak or hypoactive BIS, in turn, is theoretically associated with low anxiety, insensitivity to punishment cues, and failures in passive avoidance learning.

As related to ADHD, Quay $(1988,1997)$ proposed that weak BIS activation is central to ADHD. Low BIS activation or reactivity would impair the ability to interrupt ongoing activity and to detect and effectively respond to stimuli that signal the potential for punishment. While some studies provide support for the weak-BIS hypothesis of ADHD (e.g., Beauchaine, Katkin, Strassberg, \& Snarr, 2001; Lazzaro et al., 1999; Quay, 1997), other research has produced findings that are inconsistent with the weak-BIS model (Crone, Jennings, \& Van der Molen, 2003; Hartung, Milich, Lynam, \& Martin 2002; Iaboni, Douglas, \& Baker, 1995). Consequently, the weak-BIS hypothesis likely has limited value as a theory of ADHD, and the behavior inhibition deficits among those with ADHD are unlikely to be the sole result of punishment insensitivity and associated processes.

Within the context of Gray's theory, Newman (1987) has alternatively proposed that disinhibited behavior is largely related to the dominance of a second conceptual brain system in Gray's model, the behavioral activation (or approach) system (BAS). Gray (1987) has proposed that the BAS becomes activated in response to cues that signal reward or relief from punishment, and that BAS-dominant individuals tend to demonstrate trait-like impulsivity. According to the response modulation hypothesis by Newman and colleagues (Patterson \& Newman, 1993; Wallace, Bachorowski, \& Newman, 1991; Wallace \& Newman, 1990), disinhibited behavior is particularly likely in instances where BAS activities dominate BIS activities when both systems are activated. When this occurs among BAS-dominant or impulsive individuals, "go" or approach response sets associated with the attainment of reward predominate and are difficult to modify, even when response contingencies have shifted or become incompatible with goal-directed behavior. At the heart of this failure to appropriately adjust or regulate behavior are impairments in the regulation of attentional resources to nondominant cues that have informational value for ongoing behaviors. In the case of BAS-dominant individuals, dominant cues that primarily influence responding are those associated with reward. For such individuals, attentional resources are largely allocated to cues and behaviors associated with reward or its attainment, and attentional resources are largely unallocated to non-dominant cues that have relevance for ongoing behavior, such as those that signal potential punishment. Consequently, persons who fail to attend to nondominant cues are unlikely to have their behavior modified by them, and the dominant response set will persist even though it may no longer be effective or adaptive (MacCoon, Wallace, \& Newman, 2004; Patterson \& Newman, 1993).

In a mixed incentive context where both rewards and punishers are simultaneously contingent on behavior $(\mathrm{R}+\mathrm{P})$, Newman's model would predict that BAS dominant individuals would (a) be oriented and allocate disproportionately more ongoing attention to dominant $\mathrm{S}+$ cues, and (b) allocate disproportionately less attention to non-dominant cues, which would include $\mathrm{S}$ - stimuli and performance feedback following punishment. As a result of the combined effect of these factors, disinhibited persons compared to others would be expected to commit more passive avoidance errors (PAEs), which are commission errors characterized by the inability to withhold responses to $\mathrm{S}-$ stimuli. In such instances, BAS dominance over the BIS results in a tendency whereby responding for reward is stronger than the tendency to inhibit responding that may lead to punishment, which results in a PAE. Consistent with the response modulation hypothesis, research has demonstrated a greater tendency among disinhibited adults to make more PAEs than controls while simultaneously responding for reward (e.g., Farmer et al., 2003; Newman, Patterson, Howland, \& Nichols, 1990; Newman, Widom, \& Nathan, 1985; Patterson, Kosson, \& Newman, 1987).

One common experimental paradigm used to evaluate response modulation deficits is the go/no-go task. When mixed incentives (rewards and punishers) are contingently available for responding during the go/no-go task, a participant is challenged to maintain response performance (i.e., a "go" response or to key press in the presence of $S+$ stimuli) while 
alternative stimuli (i.e., $\mathrm{S}-$ stimuli) that require a competing motor response (a "no-go" response or the withholding of a key press) are also present. Among youth with ADHD, a number of studies have employed the go/no-go task with generally consistent findings. In Shue and Douglas (1992) and Trommer, Hoeppner, Lorber, and Armstrong (1988), for example, children diagnosed with ADHD compared to normal controls made more PAEs. In an extension of this research by Milich, Hartung, Martin, and Haigler (1994) with 90 youth between the ages of 13 and 21 with a history of behavioral or psychiatric disorders, PAE frequency was positively correlated with dimensionally-represented ADHD symptomatology but not conduct disorder (CD) symptomatology for males, whereas no significant correlation was obtained for PAE frequency and either ADHD or CD symptomatology for females. However, the correlations for females $(n=17)$ were in the predicted direction for ADHD symptomatology ( $r s=.39$ for past symptomatology, .32 for current), and similar for current CD symptomatology $(r=.32)$. Findings were inconsistent for youth in the reward-only $(\mathrm{R})$ condition. Similarly, Hartung et al. (2002) found among 172 clinic- and non-clinic referred youth between the ages of 13 to 18 years that ADHD symptoms were predictive of PAEs for both males and females, but only in the mixed incentive $(\mathrm{R}+\mathrm{P})$ condition and not the punishment-only $(\mathrm{P})$ condition. In contrast, CD symptoms were not predictive of PAEs for females in either incentive condition or males in the $\mathrm{R}$ condition, whereas it was predictive for males in the $\mathrm{R}+\mathrm{P}$ condition. Three implications can be suggested from these studies: (a) consistent with Newman's theory, PAEs are more likely to reliably emerge for disinhibited persons in the mixed incentive context $(\mathrm{R}+\mathrm{P})$ than in the case where either contingency is presented alone ( $\mathrm{R}$ or $\mathrm{P}$ ), (b) ADHD symptomatology is more consistently associated with PAEs for both boys and girls than CD symptoms, and (c) there might be some differences in the expression of behavioral disinhibition between males and females on the go/no-go task.

Other studies that have employed the go/no-go task, however, suggest that PAEs are more likely among youth with ADHD regardless of incentive condition. Iaboni et al. (1995), for example, compared 18 boys between the age of 8 and 13 years with ADHD against 18 male normal controls within the same age range, and found that those with ADHD demonstrated more PAEs in the $\mathrm{R}+\mathrm{P}$ condition as well as in the $\mathrm{R}$-only and P-only conditions. This finding was interpreted by the authors as inconsistent with Newman's response modulation hypothesis and more consistent with the notion that ADHD is defined by a generalized inhibition deficit. Similarly, Gomez (2003) utilized a motivational go/no-go task to investigate behavioral disinhibition among 30 boys with ADHD (ages 9-13) relative to normal controls. In this study, youth with ADHD were found to make more PAEs in each of three reinforcement conditions: R-only, P-only, and $\mathrm{R}+$
P. However, the most PAEs among ADHD youth were observed in the $\mathrm{R}+\mathrm{P}$ condition compared to the remaining two. This finding was interpreted as consistent with the general response inhibition deficit model as well as Newman's response modulation hypothesis.

Newman's response modulation hypothesis as applied to ADHD has been generally supported while some limitations have also been suggested. The present study consequently sought to simultaneously evaluate several predictions associated with Newman's response modulation model while at the same time controlling for the potential influence of other variables that covary with ADHD. A unique aspect of this study is that it directly evaluates the role of response reflection in relation to disinhibited behavior, something that other investigations of Newman's theory with ADHD samples have not previously explored. In relation to these general study objectives, several specific hypotheses were tested or explored, and these are delineated below.

First, the present study sought to examine whether adolescent youth diagnosed with ADHD relative to normal controls would make more PAEs in a mixed incentive $(\mathrm{R}+\mathrm{P})$ context in order to further clarify the potential utility of the response modulation hypothesis in accounting for inhibitory deficits that characterize ADHD. Consistent with predictions from the response modulation hypothesis and findings from Iaboni et al. (1995), we hypothesized that the greatest differences in PAE commission by members of the two groups would occur within the last blocks of trials. That is, those with ADHD were hypothesized to demonstrate a flatter learning curve over time, thus suggesting a comparative deficit in efficient responding to punishment signals by withholding responses when simultaneously responding for reward.

Second, there is a growing consensus that dimensional representations of disorder concepts are frequently associated with greater reliability indices, often more conceptually congruent with the population variability and continuous distribution of features that define disorders, and more appropriate for hypothesis testing than categorical representations (Farmer, 2000; Kraemer, Noda, \& O’Hara, 2004). Given this, and following the example of other ADHD researchers in this area (Hartung et al., 2002; Milich et al., 1994), we evaluated PAL when ADHD was categorically defined according to DSM diagnostic decision rules and dimensionally based on scores from a parent-rating measure of overall ADHD symptomatology.

Third, this study also evaluated the possible influence of other variables (i.e., IQ, conduct disorder/oppositional defiant disorder symptomatology, parent-rated anxiety and depression) on PAE occurrence. Given findings from Milich et al. (1994) and Hartung et al. (2002), we hypothesized that any observed differences in PAE frequency among ADHD and control groups would not be fully accounted for by $\mathrm{CD}$ and oppositional defiant disorder symptoms. 
diagnosis of ADHD were subsequently provided with a description of the present study and asked to participate. Data from three other service-referred youths were not included in the present research as these persons met diagnostic criteria for psychiatric conditions but not ADHD (i.e., bipolar II disorder, oppositional defiant disorder, and conduct disorder).

The control group ( $n=23,39.1 \%$ males) had been recruited through advertising at local schools and other community resources, and had received the same clinical evaluation as the ADHD group. Data for one additional control participant was not included in the present study due to a technical problem during the administration of the computerized PAL task. Efforts were made during participant recruitment to have approximately equal numbers of males and females in both groups. The overall sample was predominantly white and of European descent $(95.1 \%)$, with the remaining (4.9\%) of Maori descent (i.e., indigenous peoples of New Zealand).

Assessments and measures

\section{Diagnostic protocol for $A D H D$}

The Schedule for Affective Disorders and Schizophrenia for School-Age Children-Present and Lifetime Version (K-SADS-PL; Kaufman et al., 1997) was used to assess $D S M$ diagnostic concepts specific to youth. For all participants, diagnostic interviews were conducted separately with the adolescent and a parent. The long versions of the Conners' Rating Scales-Revised (CRS-R; Conners, 1997) were also used to specifically assess ADHD. This instrument provides separate rating forms for parents, teachers and adolescents. For this study, parent and adolescent ratings were considered. The parent rating involves an 80-item scale, and includes measures of oppositional behaviors, hyperactivity, other indices of ADHD, and cognitive problems. The 87-item adolescent self-report assesses the same areas as the parental scale, with the inclusion of anger control problems.

To be included in the ADHD group, a participant would have met each of the following criteria: (a) DSM-IV-TR diagnostic criteria for ADHD based on the clinician summary of the K-SADS-PL parent and adolescent interview, whereby parental report information related to the presence versus absence of externalizing symptoms would supercede the adolescent report in the event of a discrepancy, (b) a $T$-score $\geq 65$ on at least one of the ADHD subscales of the CRS-R parent form, and (c) evidence of ADHD symptoms prior to the age of seven established either through a past diagnosis of ADHD or, among new cases, through parental report and past school report cards.

To be included within the control group, an adolescent would have failed to meet ADHD criteria according to the 
K-SADS-PL. Clinic-referred participants who failed to meet inclusionary criteria for the ADHD group were not included in the control group. All clinical interviews and testing were conducted in laboratories within a department of psychology in a mid-sized university. Consent and assent forms were reviewed with both parents and adolescents prior to study participation.

Parents of children in the ADHD group who were taking psycho-stimulant medication (i.e., methylphenidate; $n=14$ or $77.8 \%$ ) were asked not to give their children this medication on the morning of testing with the interactive computer task as stimulant medications can enhance reward sensitivity (Wilkison, Kircher, McMahon, \& Sloane, 1995) and go/no-go task performance (Trommer et al., 1991). As methylphenidate has an approximate half-life of $4.5 \mathrm{~h}$ (Shader et al., 1999), a 24-hour elimination period should have ensured that the majority of the active ingredient had been eliminated prior to testing. Five (27.8\%) members of the ADHD group took at least one medication other than stimulant medication (paroxetine, clonidine, fluoxetine, citalopram), and one of the controls (4.3\%) took paroxetine. As these medications were prescribed for reasons other than ADHD, these medications were not discontinued for purposes of this research.

\section{Measures of demographic variables}

The New Zealand Socioeconomic Index of Occupational Status (NZSEI; Davis, McLeod, \& Ransom, 1997), based on 1991 New Zealand census data, was used as a measure of socio-economic status. The NZSEI scores range between 10 and 100 , with higher scores indicative of higher socioeconomic status.

\section{Estimation of intellectual functioning (IQ)}

IQ was estimated using the Block Design and Vocabulary subtests of the WISC-III (Wechsler, 1991) or the WAIS-III (Wechsler, 1997). Scores from these subtests, when combined, are good estimators of Full Scale IQ (Sattler, 2001).

\section{Assessment of $O D D$ and $C D$ symptomatology}

The K-SADS-PL (Kaufman et al., 1997) was used to assess the presence of ODD and CD symptomatology. In the present research, an aggregated variable that dimensionally represented the presence of these symptoms was derived. For each disorder concept, individual symptom presence versus absence was first determined according to DSM-IV-TR criteria. In the event a symptom was present, it was assigned a

value of 1 . Symptoms that did not reach diagnostic thresholds were assigned a value of 0 . Within each disorder concept, symptom ratings were summed and then divided by the total number of symptoms that defined the diagnostic concept. Once this was done for both ODD and CD symptomatology, the resultant proportionalized values were added together, and this sum served as the index for combined ODD/CD symptomatology.

\section{Assessment of the experience of anxiety and depression}

The Child Behavior Checklist (CBCL; Achenbach, 1991) is designed to identify children at risk for behavior problems. The Parent version of this checklist was used to assess the experience of anxiety and depression in the adolescent youth. Ratings for one control participant were not available; consequently, this individual was not included in analyses that involved this measure.

Procedure

Assessment of passive avoidance learning (PAL)

A successive go/no-go discrimination interactive computer task was used to assess PAL (Farmer et al., 2003; Patterson et al., 1987, Experiment 2). Computer task stimuli consisted of 12 two-digit numbers that were presented sequentially on a computer monitor. Six of these numbers were positive discriminative stimuli $(\mathrm{S}+)$. When a participant responded to the presence of the stimuli by depressing the space bar, he or she received immediate feedback (i.e., the word "Correct" which appeared in big blue letters across the center of the computer monitor) and was awarded with $10 \notin$ by the experimenter who placed a coin in a dish positioned next to him or her. The remaining 6 numbers were negative discriminative stimuli $(\mathrm{S}-)$. When a participant responded to these stimuli, he or she received immediate feedback (i.e., the word "Wrong" which appeared in big red letters across the center of the computer monitor). The experimenter also removed a $10 \phi$ coin from the dish. To avoid punishment (i.e., the "Wrong" feedback and the loss of $10 \phi$ ) when S - were presented, participants had to withhold responding. That is, punishment could be avoided during the task by passive avoidance. A failure to withhold responding in the presence of an $\mathrm{S}$ - signal was regarded as a passive avoidance error (PAE). If the participant did not respond to an $\mathrm{S}+$ or $\mathrm{S}-$ within a $3 \mathrm{~s}$ time period, no feedback was provided and a new trial was automatically initiated. If the participant responded to a number by pressing a key, feedback was presented for a maximum of $7 \mathrm{~s}$ or until the subject terminated it by pressing a key to initiate the next trial. The inter-trial interval between number presentations was $1 \mathrm{~s}$. All participants
429

1

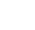

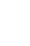

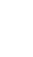
7 9

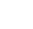

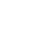
3

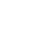

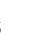

7


Table 1 Means (and standard deviations) for controls and K-SADS-PL diagnosed youth with ADHD on parent- and self-reported ADHD symptomatology as assessed by the CRS-R

\begin{tabular}{lllllr}
\hline CRS-R scale & ADHD & Controls & $t$ & $p$ & $d$ \\
\hline Parent report & & & & & \\
$\quad$ CRS-R inattentive & $75.72(9.98)$ & $49.04(10.20)$ & 8.43 & $<.001$ & 1.60 \\
$\quad$ CRS-R hyperactive-impulsive & $74.56(10.55)$ & $52.09(9.69)$ & 7.09 & $<.001$ & 1.49 \\
$\quad$ CRS-R total & $77.94(8.90)$ & $50.43(10.55)$ & 8.86 & $<.001$ & 1.63 \\
Adolescent self-report & & & & & \\
$\quad$ CRS-R inattentive & $57.11(10.39)$ & $44.04(8.93)$ & 4.33 & $<.001$ & 1.13 \\
$\quad$ CRS-R hyperactive-impulsive & $52.50(11.18)$ & $43.00(8.84)$ & 3.04 & .004 & .87 \\
CRS-R total & $52.72(10.65)$ & $42.48(9.94)$ & 3.18 & .003 & .90 \\
\hline
\end{tabular}

Notes. K-SADS-PL = Schedule for affective disorders and schizophrenia for school-age children-present and lifetime version; ADHD = Attention-deficit/hyperactivity disorder; CRS-R = Conners' rating scales-revised.

The primary dependent variable from the computer task was the number of PAEs (i.e., responding rather than inhibiting a response to an $\mathrm{S}-$ ) committed during treatment trials. In secondary analyses, the number of omission errors (OEs; instances of non-responding to an $\mathrm{S}+$ ) committed during treatment trials was also evaluated. This study also considered the role that reflection on task performance (i.e., the median amount of time, in milliseconds, that subjects viewed response-contingent feedback) had on actual task performance, as well as associations that reflection on response feedback had with group membership.

\section{Results}

Preliminary analyses

Participant characteristics as a function of group membership

A series of preliminary analyses examined the distribution of demographic characteristics in relation to group membership. The sex distribution was similar among the ADHD and control groups, $X^{2}(1, N=41)=1.10, n s$. Similarly, no age differences were noted as a function of group, $t(39)=0.53$, nor were any significant differences noted in socioeconomic status, $t(38)=0.17$. The mean socioeconomic status of the sample was $56.30(S D=20.67)$, which is indicative of middle socio-economic status.

As would be expected, the control and ADHD groups significantly differed on indices of self- and parentreported ADHD symptomatology as assessed by the CRS-R (Table 1). Consistent with previous research (e.g., Smith, Pelham, Gnagy, Brooke, \& Evans, 2000), youth with ADHD under-reported instances of overactivity and inattention relative to parents. The control and ADHD groups also differed on estimated full scale IQ, $t(39)=2.77, p<.01$, 
$d=.89$. Those with ADHD had an estimated IQ within the normal range $(M=97.89, S D=14.07)$; however, this mean IQ was significantly less than that of controls $(M=108.91$, $S D=11.40)$. Similarly, there was a significant group difference on parent-rated anxiety and depression on the CBCL, $t(38)=4.83, p<.001, d=1.61$. Parents rated youth with ADHD as experiencing more anxious and depressed symptoms than controls ( $M \mathrm{~s}=60.78$ and 50.27, respectively). Consequently, a portion of the analyses presented below examined the potential influence of IQ and symptoms of anxiety and depression on PAL.

Within the ADHD group, 10 persons (50\% male) were classified as predominantly inattentive and 8 persons $(62.5 \%$ male) as combined inattentive and hyperactive-impulsive. No persons within the ADHD group were classified as predominantly hyperactive-impulsive. For both self- and parent-reported scales on the CRS-L, only one difference was apparent between the inattentive and combined groups, and this was for the parent-rated impulsivity-hyperactivity scale, $t(16)=2.22, p<.05, d=.53$. As would be expected, youth classified as predominantly inattentive had lower scores on this scale $(M=70.10)$ than those in the combined group $(M=80.13)$. When the sex distributions within the inattentive and combined groups were compared, no departures from expectation were observed, $X^{2}(1, \mathrm{~N}=18)=.28$.

\section{Median reaction time to task stimuli}

When ADHD and control groups were compared on reaction time following the presentation of task stimuli (both $\mathrm{S}+$ and $S-$ ), no significant differences emerged, $t(39)=0.57$, $n s$. This finding suggests that there was no overall speedaccuracy trade-off pattern that operated differently as a function of group membership.

\section{Manipulation checks}

\section{$P A L$}

A repeated-measures ANOVA was performed with trial block as the within-subjects factor and PAE frequency as the dependent measure to explore whether the PAL manipulation was successful. When both control and ADHD-diagnosed participants were included in the same analysis, a significant effect for trial block was obtained, $F(2,80)=38.78$, $p<.001, \eta^{2}=.49$. Post hoc analyses indicated that PAE means for each trial block were significantly different at $p<.001$ : Block $1=8.95$, Block $2=6.12$, Block $3=4.90$. Participants, on average, reduced the number of PAEs committed over consecutive sets of trials as a result of learning to avoid punishment by withholding responses to $S-$ stimuli. This finding supports the validity of the PAL experimental procedure for the entire sample.

\section{Omission errors (OEs)}

When OEs served as the dependent variable, a 2 (group) $\times$ 3 (trial block) mixed-model ANOVA revealed an absence of any significant main or interaction effects. Consistent with previous research, the mean number of OEs committed by participants during all non-baseline trials was relatively small $(M=10.61, S D=8.17)$. These findings suggest that the baseline manipulation to create a dominant approach response set for responding to task stimuli was successful, and the absence of group differences suggests that members of both groups were equally attentive during the PAL computer task (Trommer et al., 1988).

Primary analysis: passive avoidance learning (PAL) as a function of group membership

Frequency of passive avoidance errors (PAES) as a function of group membership and learning trials

Table 2 presents mean PAE frequencies as a function of group and trial block. A 2 (group) $\times 3$ (trial block) mixedmodel ANOVA revealed a significant main effect for trial block, $F(2,78)=40.54, p<.001, \eta^{2}=.51$ (Ms: Block $1=8.95$, Block $2=6.12$, Block $3=4.90$ ). Paired $t$-tests revealed that the difference in the mean number of PAEs between Block1 and Block 2 was significant, $t(40)=4.78, p<$ $.001, d=1.51$, as was the difference between Block 1 and Block $3 t(40)=9.03, p<.001, d=2.86$. Similarly, significant differences were apparent between Block 2 and Block $3, t(40)=3.57, p<.001, d=1.13$.

A significant main effect for group on PAE frequency was also obtained, $F(1,39)=5.50, p<.05, \eta^{2}=.12$. Participants with ADHD committed more PAEs on average within trial blocks than controls $(M \mathrm{~s}=8.04$ and 5.58, respectively). A significant effect was also observed for the group by trial block interaction, $F(2,78)=7.65$, $p<.01, \eta^{2}=.16$. Post-hoc analyses revealed that the ADHD group did not significantly differ from controls on the average number of PAEs committed during Block $1(M \mathrm{~s}=9.22$ and 8.74, respectively). However, for Block

Table 2 Means for PAE frequency as a function of group and trial block

\begin{tabular}{lllc}
\hline \multirow{3}{*}{ Group } & \multicolumn{3}{l}{ PAE means } \\
\cline { 2 - 4 } & Block 1 & Block 2 & Block 3 \\
\hline All participants $(n=41)$ & 8.95 & 6.12 & 4.90 \\
ADHD $(n=18)$ & 9.22 & 8.17 & 6.72 \\
Controls $(n=23)$ & 8.74 & 4.52 & 3.48
\end{tabular}

Notes. $\mathrm{PAE}=$ passive avoidance error, $\mathrm{ADHD}=$ attentiondeficit/hyperactivity disorder. 
2 , there was a significant difference between groups, $t(39)=2.95, p<.01, d=.95$, whereby those with ADHD made more PAEs than controls $(M \mathrm{~s}=8.17$ and 4.52 , respectively). Similarly, there was a significant difference between ADHD participants and controls in PAE frequency during Block 3, $t(39)=2.95, p<.01, d=.95$, whereby those with ADHD $(M=6.72)$ committed significantly more PAEs than controls $(M=3.48)$. Relative to controls, PAL across trials was relatively modest for ADHD participants (Table 2). ${ }^{2}$

\section{Covariance analyses}

Given a number of possible variables that covary with ADHD and might influence PAL, three covariance analyses were performed. In each instance, the primary analysis was repeated, with the exception that one of three variables was first considered as a covariate (i.e., IQ, ODD/CD symptoms, and parent-rated anxiety and depression).

\section{Group membership and PAE frequency within trial blocks after controlling for the influence of IQ}

Given the significant difference in IQ observed as a function of group membership, correlational analyses were first performed to determine if an association also existed between IQ and PAL. For the overall sample, a negative significant correlation for IQ and total number of PAEs across trial blocks was observed $(r=-.48, p<.01)$. When correlations were computed that examined the strength of the association between IQ and PAE frequency within trial blocks, stronger associations were evident for the last two trial blocks ( $r \mathrm{~s}$ : Block $1=-.29, p<.07$; Block $2=-.52, p<.001$; Block $3=-.45, p<.01)$.

The main analysis that examined PAEs as a function of group and trial block was repeated, this time with IQ as a covariate. Once the influence of IQ was statistically controlled, no significant effect remained for group, $F(1,38)=1.54$, or for trial block, $F(2,76)=1.07$. However, the group by trial block interaction remained significant, $F(2,76)=5.01$, $p<.01, \eta^{2}=.12$. Planned post hoc comparisons revealed that there was no significant group difference for Block 1, $t(39)=0.35$. However, trends with associated medium effect sizes were noted for Blocks 2 and 3, whereby those with ADHD committed more PAEs after controlling for IQ than

\footnotetext{
${ }^{2}$ Five of the control participants had total ADHD scores on the parentrated CRS-R at or above a T-score of 60 . Given the possible presence of subthreshold ADHD among these five persons, this analysis was rerun with these five control participants excluded. The obtained results were highly similar, whereby the effect for trail block was significant, $F(2,68)=38.15, p<.001, \eta^{2}=.53$, as was the main effect for group, $F(1,34)=4.22, p<.05, \eta^{2}=.11$, and the group by trial block interaction, $F(2,68)=14.87, p<.001, \eta^{2}=.29$.
}

controls: Block 2: $t(39)=1.76, p<.09, d=.58$; Block 3: $t(39)=1.94, p<.06, d=.63$.

Group membership and PAE frequency within trial blocks after controlling for the influence of $O D D$ and CD symptomatology

Correlational analyses were first performed to determine if associations existed between PAE frequency and ODD/CD symptomatology. For the overall sample, a positive trend was noted $(r=.28, p<.10)$. When correlations were computed that examined the strength of the association between PAE frequency and ODD/CD symptomatology separately for each trial block, a significant association was observed only for the last trial block ( $r$ : Block $1=.17$, $n s$; Block $2=.25, n s ;$ Block $3=.34, p<.05)$.

When the proportion of concurrent ODD/CD symptomatology was used as a covariate, no significant effect remained for group, $F(1,38)=2.23$. A significant effect for trial block, however, was observed, $F(2,76)=22.32, p<.001, \eta^{2}=.37$. Adjusted PAE means for trial blocks were 8.93, 6.35, and 5.07 for Blocks 1, 2, and 3, respectively. Each adjusted block mean was significantly different from the other at $p<.001$.

The group by trial block interaction was also significant, $F(2,76)=7.57, p<.01, \eta^{2}=.17$. Post hoc comparisons revealed that there was no significant group difference for Block 1, $t(39)=.30$. A significant difference was noted, however, for Block 2, $t(39)=2.35, p<.05, d=.76$. For Block 3, there was a trend with an associated medium effect size whereby those with ADHD committed more PAEs after controlling for ODD/CD symptomatology than controls, $t(39)=1.92, p<.07, d=.62$.

\section{Group membership and PAE frequency within trial} blocks after controlling for the influence of anxiety and depression

Correlations were first computed to evaluate the association between overall PAE frequency and parent-rated anxious/depressed symptomatology. For both groups combined, a significant positive association was observed $(r=.35, p$ $<.05)$. When correlations were computed to evaluate the strength of the association between PAE frequency and anxious/depressed symptomatology for each trial block, stronger associations were evident for the last two blocks ( $r$ s: Block $1=.16, n s ;$ Block $2=.31, p<.05$; Block $3=.46$, $p<.01)$.

When the proportion of parent-rated anxious/depressed symptomatology was used as a covariate, no significant effect remained for group, $F(1,37)=2.29$. A trend for trial block, however, was observed, $F(2,74)=2.66, p<.08, \eta^{2}=.07$. Adjusted PAE means for trial blocks were 8.88, 5.95, and 
4.80 for Blocks 1, 2, and 3, respectively. Post-hoc analyses revealed that the difference in means between Blocks 1 and 2 were marginally different, $t(39)=1.97, p<.06, d=.63$, as was the difference between Blocks 2 and 3, $t(39)=1.79, p$ $<.09, d=.57$, with both contrasts associated with medium effect sizes. The difference between Blocks 1 and 3 was significant, $t(39)=4.18, p<.001, d=1.34$.

The group by trial block interaction was also significant, $F(2,74)=6.62, p<.01, \eta^{2}=.15$. Post hoc comparisons revealed that there was no significant group difference for Block 1, $t(38)=0.08$, or for Block $3, t(38)=1.60$. A significant difference, however, was noted for Block $2, t(38)=2.56$, $p<.05, d=.83$, whereby those with ADHD committed more PAEs after controlling for anxious/depressed symptomatology than controls. ${ }^{3}$

\section{Group membership, PAE frequency and reflection on} PAE response feedback

For all participants regardless of group membership, the correlation between the number of PAEs committed across trials and the median reflection time on response feedback following PAEs was $-.45(p<.01)$. This indicates that fewer PAEs were observed if participants spent more time viewing PAE response feedback. This finding raises the possibility that significant group effects in relation to PAE were influenced by the tendency to stop and pause following punishment. To further evaluate this possibility, a set of analyses was first undertaken that involved the examination of whether reflection of response feedback predicted PAE frequency in each trial block. Three separate regression analyses were performed, whereby PAE frequency for a given trial block served as the predicted variable. For each analysis, the median reflection time following reward feedback was entered as the first predictor (to control for overall rapid response style; see Patterson et al., 1987, p. 571) followed by median reflection time

\footnotetext{
${ }^{3}$ It is possible that adolescents might be more accurate reporters of internalizing experiences than parents. Consequently, this analysis was rerun, with youth-rated anxious and depressed symptoms on the CRS-R (Conners, 1997) used as the covariate among participants for whom such data were available ( $n=21$ for controls, $n=17$ for the ADHD group). No significant effect was obtained for trial block, $F(2$, $70)=1.85, n s, \eta^{2}=.05$. A significant trend $(p<.08)$ was obtained for group, $F(1,35)=3.48, \eta^{2}=.09$, whereby those with ADHD committed more PAEs than controls (adjusted means: 7.80 and 5.67, respectively). There was also a significant trial block by diagnosis interaction, $F(2$, $70)=6.23, p<.01, \eta^{2}=.18$. Planned contrasts of group means within each trial block indicated that groups did not differ in PAE frequency in Block 1, $t(36)=.18$, but did differ in Block 2, $t(36)=2.55, p<$ $.05, d=.80$, and Block 3, $t(36)=2.29, p<.05, d=.72$. In both of these latter two instances, those with ADHD committed more PAEs than controls (adjusted means: 7.76 and 4.34, respectively, for Block 2; 6.41 and 3.67, respectively, for Block 3). Parent- and youth-rated anxious/depressed symptoms were moderately correlated in this sample $(r=.55, p<.001)$.
}

following punishment feedback entered in the second step. In none of these analyses did reflection on reward emerge as a significant predictor. However, in each instance, reflection on punishment feedback predicted PAE frequency after the influence of reflection on reward was removed. Reflection on punishment feedback significantly predicted PAEs during Block $1\left[F(1,38)=15.03, p<.001, \Delta R^{2}=.28\right]$, Block $2\left[F(1,38)=12.36, \Delta R^{2}=.24, p<.001\right]$, and Block $3\left[F(1,38)=7.33, p<.01, \Delta R^{2}=.16\right]$. Findings from these analyses suggest that pausing and reflecting following punishment, not an overall rapid response tendency per se, was significantly and substantially related to PAE commission.

Given these findings, we again examined PAL as a function of group (i.e., ADHD vs. control), this time with reflection on punishment as a covariate, as the above findings raise the possibility that the effects that involve the group variable may be entirely due to response reflection tendencies following punishment feedback. When this was done, a significant effect for the covariate was observed, $F(1,38)=13.40, p<.001, \eta^{2}=.26$. There was also a significant effect for group, $F(1,38)=8.60, p<.01, \eta^{2}=.18$. Those with ADHD committed more PAEs than controls (adjusted means: 8.16 for ADHD, 5.48 for controls). There was also a significant within-subjects effect for trial block, $F(2$, $76)=14.68, p<.001, \eta^{2}=.28$, and a significant trial block by group interaction, $F(2,76)=7.43, p<.001, \eta^{2}=.16$. For the within-subjects main effect, adjusted means for each trial block were significantly different at $p<.05$ : Block $1=9.00$, Block $2=6.36$, Block $3=5.11$. Planned contrasts related to the trial block by group interaction revealed that within Block 1 there was no significant difference between groups, $t(39)=.72$, ns. However, for Block 2 , there was a significant difference in PAE commission between groups, $t(39)=3.51, p<.001, d=.91$, whereby those with ADHD committed more PAEs than controls (adjusted means: 8.30 and 4.41, respectively). Similarly, there was a significant difference in Block 3 PAEs as a function of group, $t(39)=3.35, p<.01, d=.90$, with the ADHD group committing more PAEs than controls (adjusted means: 6.83 and 3.40 , respectively). Overall, the group and group by trial block interaction effects observed in our primary analysis were preserved even after covarying out the influence of reflection on punishment feedback. These findings suggest that ADHD diagnostic status and reflection on punishment feedback are significantly and independently related to PAE commission.

Association of reflectivity with other relevant variables

Correlations were also computed to investigate the degree of association between reflection on response feedback following PAEs and other variables related to PAEs in this study. The amount of time spent reflecting on punishment 
Table 3 Correlations of parent-reported ADHD symptomatology with PAE frequency for combined sample $(n=41)$

Notes. CRS-R $=$ Conners' Rating Scales-Revised; ADHD $=$ attention-deficit/hyperactivity disorder; PAEs $=$ passive avoidance errors.

${ }^{* * *} p<.001,{ }^{* *} p<.01,{ }^{*} p<$ $.05,^{\dagger} p<.10$.

\begin{tabular}{|c|c|c|c|c|}
\hline \multirow[b]{2}{*}{ CRS-R Scale } & \multirow{2}{*}{$\begin{array}{l}\text { For all non-baseline } \\
\text { trians }\end{array}$} & \multicolumn{3}{|c|}{ Total number of PAEs } \\
\hline & & Block 1 & Block 2 & Block 3 \\
\hline \multicolumn{5}{|l|}{ Bivariate correlations } \\
\hline Inattentive & $.32 *$ & .05 & $.37^{*}$ & $.42^{* *}$ \\
\hline Hyperactive-impulsive & .24 & -.04 & $.30^{\dagger}$ & $.35^{*}$ \\
\hline Total & $.28^{\dagger}$ & .01 & $.35^{*}$ & $.39^{*}$ \\
\hline \multicolumn{5}{|c|}{$\begin{array}{l}\text { Partial correlations controlling for } \\
\text { reflection on punishment feedback }\end{array}$} \\
\hline Inattentive & $.42^{* *}$ & .13 & $.45^{* *}$ & $.49^{* * *}$ \\
\hline Hyperactive-impulsive & $.33^{*}$ & .03 & $.38^{*}$ & $.42^{* *}$ \\
\hline Total & $.39^{*}$ & .08 & $.44^{* *}$ & $.46^{* *}$ \\
\hline
\end{tabular}

feedback was independent of estimated IQ $(r=.04, n s)$, the sum of proportionalized ODD/CD symptoms $(r=-.06$, $n s$ ), and parents' ratings of anxious and depressed moods $(r=.06, n s)$ for the sample as a whole.

Dimensional relations among PAEs, ADHD

symptomatology, and reflection on punishment feedback

Until now, we have examined PAL in relation to categorically defined ADHD, whereby contrast groups were based on whether the participant met DSM ADHD diagnostic criteria. In the analyses presented in this section, ADHD symptom features as indexed by parent ratings on the CRS-R for the entire sample $(n=41)$ were used as the index of overall ADHD.

As evident in Table 3, the associations between PAEs and ADHD symptomatology were evident in the last two blocks of learning trials but not during the first trial block. Similar patterns of correlations were obtained for both inattentive and hyperactive-impulsive features. When partial correlations were computed that controlled for reflection on punishment feedback, the pattern of correlations was quite similar to that displayed in the bivariate correlations (Table 3). Additionally, reflection on punishment feedback did not significantly correlate with CRS-R parent-rated inattentive $(r=.12)$, impulsive-hyperactive $(r=.13)$, and total ADHD $(r=.12)$ scales. These findings are similar to those obtained when ADHD was treated as a categorical variable (present versus absent according to diagnostic criteria). Dimensionally represented ADHD symptomatology has moderate associations with PAE commission in later learning trials, and this association is independent of the tendency to reflect on punishment feedback.

\section{Discussion}

Findings from this study provide additional support for the view that impairments in behavioral inhibition constitute a central feature of ADHD. In a mixed incentive context where both reward and punishment contingencies were simultaneously operative $(\mathrm{R}+\mathrm{P})$, adolescents with ADHD committed more PAEs overall than controls, particularly in the latter trial blocks. This finding suggests that members of the control group were able to adjust their dominant goal-directed response set to accommodate stimulus cues that signaled punishment under some conditions. In contrast, youth with ADHD displayed greater difficulty withholding responding to $\mathrm{S}$ - stimuli, as evident by relatively flat learning curves relative to controls. This impairment in behavior shifting from activity to passivity in accordance with alternating contingencies among youth with ADHD is consistent a central postulate of Newman's response modulation hypothesis as it applies to disinhibited persons.

Covariance analyses that examined the potential influence of IQ, ODD/CD symptoms, and the experience of anxious and depressed mood indicated that differences between ADHD and control groups in PAE frequency generally remained even when common variance associated these variables was statistically removed. The observed inverse association between IQ and PAE frequency is consistent with findings reported in Hartung et al. (2002). While the control of IQ as a possible influence resulted in somewhat weaker findings, a significant group by trial block interaction was still observed. Additionally, although ADHD and ODD/CD syndromes demonstrate significant comorbidity, findings from this study as well as others (Hartung et al., 2002, Milich et al., 1994) suggest that failures to withhold responses to $\mathrm{S}$ - stimuli continue to be associated ADHD symptomatology even when the influence of ODD/CD symptomatology has been considered and removed, and provide additional support for the view that processes associated with disinhibition among those with ADHD and CD/ODD may differ in important respects (Nigg, 2000).

Another prediction from the response modulation hypothesis, that disinhibited persons reflect less on punishment feedback than others, received equivocal support. A moderate negative correlation was obtained for all participants 
that demonstrated reflection on punishment was inversely associated with PAE frequency. Learning to paus following punishment was, therefore, associated with fewer PAEs overall. When ADHD was analyzed as a diagnostic category as well as a dimensional construct, however, both reflection on punishment feedback and ADHD were independently associated with PAE commission. Furthermore, reflection on punishment feedback did not have significant associations with IQ, CD/ODD symptomatology, or anxious/depressed mood. These and similar findings (Gremore et al., 2005; Farmer et al., 2003; Patterson et al., 1987) suggest that the tendency to reflect on punished behavioral outcomes has considerable relevance in theoretical accounts of processes involved in learning to avoid punishment. However, these findings also imply that the failures in the tendency to stop and pause following punishment are largely independent of and not antithetical to hyperactive-impulsive behavioral patterns and disinhibited personality styles, and may reflect an important individual difference variable in its own right.

The exact role that reflectivity may have in relation to the attenuation of impulsive behavior remains unclear. Selfdirected speech constitutes an important aspect of reflection, whereby such self-talk provides "a means for reflection, description and self-questioning through language, creating an important source of problem-solving ability as well as a means of formulating rules and plans" (Barkley, 1997, p. 74). A central concept associated with this process, as described by Barkley (1997) and elaborated in Hayes (1989), is rule-governed behavior. A rule is a verbal description of a behavioral contingency that specifies a response or behavior, an outcome or consequence associated with that behavior, and/or an antecedent condition in the presence of which the behavior will produce the specified outcome (Anderson, Hawkins, Freeman, \& Scotti, 2000). Rulegoverned behavior, then, is a term used to denote those behaviors that are influenced by verbal statements, or rules, that specify the operating contingencies associated with behavior, and is usually used to account for behavior that is influenced by delayed consequences (Malott, Malott, \& Trojan, 2000).

Reflection on responses in terms of their associated consequences may facilitate the rule-generation process, or contribute to the refinement and accuracy of rules. In the absence of rules for behavior, behavior may have a random, trial-and-error quality that is largely influenced by immediate events, thus leading to more variable and ineffective responding (Barkley, 1997). Existent research suggests that rule use among children is associated with greater sensitivity to response feedback and less perseverative behavior (Zelazo, Reznick, \& Piñon, 1995).

It may be that the behavior of youth with ADHD is more strongly influenced by immediate environmental contingencies than rules, and that any co-occurring deficits associated with response reflection may further contribute to a relative insensitivity to punishment contingencies and account for the greater amount of variability observed in their behavior (Johansen, Aase, Meyer, \& Sagvolden, 2002). Interestingly, studies with disinhibited adults have demonstrated that task performance improves when participants are forced to temporarily suspend ongoing behavior and reflect on response feedback (Arnett, Howland, Smith, \& Newman, 1993; Newman et al., 1985; Patterson et al., 1987). These findings suggest reflection on behavioral outcomes is a skill that can be learned, and that behavioral disinhibition associated with ADHD can potentially be mitigated to some degree if the child or adolescent is successful in applying a "stop, pause, and reflect" rule before engagement in further ongoing behavior.

Findings and conclusions associated with this research should be considered along with some caveats. For example, the sizes of the ADHD and control samples were relatively small, which may have accounted for some of the insignificant trends that were observed. A related consideration is that a number of planned contrasts were performed in the course of data analyses without the application of corrections on the critical alpha levels to reduce family-wise Type I error rates. The application of such corrections would have resulted in a loss of statistical power (Keppel, 1991). Because of already existent concerns about power related to the relatively small sample size, and the increased likelihood of committing a Type II error, we elected not to perform such corrections. Consequently, analyses that yielded marginal effects, most notably the covariance analyses that controlled for the possible influence of IQ, ODD/CD symptomatology, and anxiety/depression, should be regarded with a degree of caution.

One control participant and five of the youth diagnosed with ADHD and were maintained on non-stimulant psychotropic medication that could not be ethically discontinued for purposes of this study. The extent to which such medications interacted with study variables cannot be determined. Control participants and those with ADHD were also recruited from different sources (local schools and a service agency, respectively), thus raising the possibility that group differences, when observed, might be related to referral source rather than diagnostic status.

It also is possible that non-task related behaviors were responsible for group differences in PAE frequency. It has been observed, for example, that youth with ADHD will often report high rates of task-irrelevant thoughts during experimental procedures (Shaw \& Giambra, 1993), and that such processes may account for observed group differences in PAE frequency. Irrelevant thinking, however, is unlikely to be strongly associated with PAEs in the present research, as the rates of OEs among ADHD and control youth were approximately the same. For both groups, the rate of OEs was relatively low, which suggests that both groups were equally 
engaged in at least some aspects of the experimental procedures used in the present study (Trommer et al., 1988). An absence of differences in OEs, but not PAEs, has also been observed in other studies with disinhibited adults (Farmer et al., 2003; Newman et al., 1985; Patterson et al., 1987) as well as among children with ADHD (Gomez, 2003; Hartung et al., 2002; Iaboni et al., 1995; Trommer et al., 1991).

In addition to exploring processes and correlates associated with response reflection, future studies in this area might examine various aspects associated with reinforcement contingencies in order to isolate trends responsible for group differences in task performance. This study, for example, utilized monetary incentives for performance, and the amount awarded or lost for correct and incorrect responding, respectively, was relatively modest (see Footnote 1) when compared to that used in other studies (Hartung et al., 2002; Milich et al., 1994). Given Slusarek et al. (2001) demonstrated that behavior of children with ADHD relative to controls is more equal under high intensity than low intensity reinforcement conditions, it is possible that more modest reinforcers may have produced even more discrepant outcomes between the two groups. Consequently, future studies might vary the size or nature of reinforcers, as variations in reinforcer intensity may be associated with different outcomes, as would be predicted by a number of theories on ADHD (Luman, Oosterlaan, \& Sergeant, 2005). Additionally, individuals with ADHD are recognized as a heterogeneous group (e.g., Lilienfeld \& Waldman, 1990), as reflected by distinct diagnostic subtypes (American Psychiatric Association, 2000) and varied patterns of comorbidity with other conditions (Biederman, Newcorn, \& Sprich, 1991). Future research might therefore explore differences in task performance with consideration given to these heterogeneous features, as the presence of such features may differentially influence PAL and associated processes.

Acknowledgements This research was partially supported by Research Grants U6497 and U6520 from the University of Canterbury. The authors extend their gratitude to Lorinda Field for writing the computer program used in this study, and to Tamara Clancy, Paula Bateup, Fiona Prest, and Nicola Ward for assistance with data collection, to Jacqueline Harris for assistance with data entry, and to Janice Howard and Alex Chapman for comments on an earlier draft of this report. The authors also wish to thank the families that participated in this research and the Youth Specialty Service of the Canterbury District Health Board for project support and assistance with referrals.

\section{References}

Achenbach, T. M. (1991). Manual for the child behavior checklist: 4-18 and 1991 profile. Burlington: University of Vermont.

American Psychiatric Association (2000). Diagnostic and statistical manual of mental disorders (4th ed., text rev.). Washington, DC: Author.
Anderson, C. M., Hawkins, R. P., Freeman, K. A., \& Scotti, J. R. (2000). Private events: Do they belong in a science of human behavior? The Behavior Analyst, 23, 1-10.

Arnett, P. A., Howland, E. W., Smith, S. S., \& Newman, J. P. (1993). Autonomic responsivity during passive avoidance in incarcerated psychopaths. Personality and Individual Differences, 14, 173185.

Barkley, R. A. (1997). Behavioral inhibition, sustained attention, and executive functions: Constructing a unifying theory of ADHD. Psychological Bulletin, 121, 65-94.

Beauchaine, T. P., Katkin, E. S., Strassberg, Z., \& Snarr, J. (2001). Disinhibitory psychopathology in male adolescents: Discriminating conduct disorder from attention-deficit/hyperactivity disorder through concurrent assessment of multiple autonomic states. Journal of Abnormal Psychology, 110, 610624.

Biederman, J., Newcorn, J., \& Sprich, S. (1991). Comorbidity of attention deficit hyperactivity disorder with conduct, depressive, anxiety, and other disorders. American Journal of Psychiatry, 148, 564-577.

Conners, C. K. (1997). Conners' Rating Scales-Revised: Technical manual. New York: Multi-Health Systems Inc.

Crone, E. A., Jennings, J. R., \& van der Molen, M. W. (2003). Sensitivities to interference and response contingencies in attentiondeficit/hyperactivity disorder. Journal of Child Psychology and Psychiatry, 44, 214-226.

Davis, P., McLeod, K., \& Ransom, M. (1997). The New Zealand socioeconomic index of occupational status (NZSEI). Wellington: Statistics New Zealand.

Doob, L. W. (1990). Hesitation: Impulsivity and reflection. New York: Greenwood Press.

Farmer, R. F. (2000). Issues in the assessment and conceptualization of personality disorders. Clinical Psychology Review, 20, 823851.

Farmer, R. F., Field, C. E., Gremore, T. M., Chapman, A. L., Nash, H. M., \& Mayer, J. L. (2003). Passive avoidance learning among females as a function of Cloninger's temperament typology. Personality and Individual Differences, 34, 983997.

Gomez, R. (2003). Underlying processes in the poor response inhibition of children with attention deficit/hyperactivity disorder. Journal of Attention Disorders, 6, 111-122.

Gray, J. A. (1970). The psychophysiological basis of introversionextraversion. Behavior Research and Therapy, 8, 249-266.

Gray, J. A. (1987). The psychology of fear and stress (2nd ed.). New York: Cambridge University Press.

Gremore, T. M., Chapman, A. L., \& Farmer, R. F. (2005). Anxiety, impulsivity, and behavioral disinhibition among female inmates. Personality and Individual Differences, 39, 925-936.

Hartung, C. M., Milich, R., Lynam, D. R., \& Martin, C. A. (2002). Understanding the relations among gender, disinhibition, and disruptive behavior in adolescents. Journal of Abnormal Psychology, $111,659-664$.

Hayes, S. C. (Ed.). (1989). Rule-governed behavior: Cognition, contingencies, and instructional control. New York: Plenum.

Iaboni, F., Douglas, V. I., \& Baker, A. G. (1995). Effects of reward and response costs on inhibition in ADHD children. Journal of Abnormal Psychology, 104, 232-240.

Johansen, E. B., Aase, H., Meyer, A., \& Sagvolden, T. (2002). Attention-deficit/hyperactivity disorder (ADHD) behaviour explained by dysfunctioning reinforcement and extinction processes. Behavioural Brain Research, 130, 37-45.

Kagan, J. (1966). Reflection-impulsivity: The generality and dynamics of conceptual tempo. Journal of Abnormal Psychology, 71, 1724. 
Kaufman, J., Birmaher, B., Brent, D., Rao, U., Flynn, C., Moreci, P., Williamson, D., \& Ryan, N. (1997). Schedule for affective disorders and schizophrenia for school-age children-present and lifetime version (K-SADS-PL): Initial reliability and validity data. Journal of the American Academy of Child and Adolescent Psychiatry, 36, 980-987.

Keppel, G. (1991). Design and analysis: A researcher's handbook (3rd ed.). Englewood Cliffs, NJ: Prentice Hall.

Kraemer, H. C., Noda, A., \& O'Hara, R. (2004). Categorical versus dimensional approaches to diagnosis: Methodological challenges. Journal of Psychiatric Research, 38, 17-25.

Lazzaro, I., Gordon, E., Li, W., Lim, C. L., Plahn, M., Whitmont, S., Clark, S., Barry, R. J., Dosen, A., \& Meares, R. (1999). Simultaneous EEG and EDA measures of adolescent attention deficit hyperactivity disorder. International Journal of Psychophysiology, 34, 123-134.

Luman, M., Oosterlaan, J., \& Sergeant, J. A. (2005). The impact of reinforcement contingencies on $\mathrm{AD} / \mathrm{HD}$ : A review and theoretical appraisal. Clinical Psychology Review, 25, 183-213.

MacCoon, D. G., Wallace, J. F., \& Newman, J. P. (2004). Selfregulation: Context-appropriate balanced attention. In R. F. Baumeister \& K. D. Vohs (Eds.), Handbook of self-regulation: Research, theory, and application (pp. 422-444). New York: Guilford.

Malott, R. W., Malott, M. E., \& Trojan, E. A. (2000). Elementary principles of behavior (4th ed.). Upper Saddle River, NJ: PrenticeHall.

Milich, R., Hartung, C. M., Martin, C. A., \& Haigler, E. D. (1994). Behavioral disinhibition and underling processes in adolescents with disruptive behavior disorders. In D. K. Routh (Ed.), Disruptive behavior disorders in childhood (pp. 109-138). New York: Plenum.

Newman, J. P. (1987). Reaction to punishment in extraverts and psychopaths: Implications for the impulsive behavior of disinhibited individuals. Journal of Research in Personality, 21, 464480.

Newman, J. P., Patterson, C. M., Howland, E., \& Nichols, S. L. (1990). Passive avoidance in psychopaths: The effects of reward. Personality and Individual Differences, 11, 1101-1114.

Newman, J. P., Widom, C. S., \& Nathan, S. (1985). Passive avoidance in syndromes of disinhibition: Psychopathy and extraversion. Journal of Personality and Social Psychology, 48, 1316-1327.

Nigg, J. T. (2000). On inhibition/disinhibition in developmental psychopathology: Views from cognitive and personality psychology and a working inhibition taxonomy. Psychological Bulletin, 126, $200-246$.

Oosterlaan, J., Logan, G. D., \& Sergeant, J. A. (1998). Response inhibition in $\mathrm{AD} / \mathrm{HD}, \mathrm{CD}$, comorbid $\mathrm{AD} / \mathrm{HD}+\mathrm{CD}$, anxious, and control children: A meta-analysis of studies with the stop task. Journal of Child Psychology and Psychiatry, 39, 411-425.

Oosterlaan, J., \& Sergeant, J. A. (1996). Inhibition in ADHD, aggressive, and anxious children: A biologically-based model of child psychopathology. Journal of Abnormal Child Psychology, 26, 161-174.

Patterson, C. M., Kosson, D. S., \& Newman, J. P. (1987). Reaction to punishment, reflectivity, and passive avoidance learning in extroverts. Journal of Personality and Social Psychology, 52, 565-575.
Patterson, C. M., \& Newman, J. P. (1993). Reflectivity and learning from aversive events: Towards a psychological mechanism for the syndromes of disinhibition. Psychological Review, 100, 716-736.

Quay, H. C. (1988). Attention deficit disorder and the behavioral inhibition system: The relevance of the neuropsychological theory of Jeffrey A. Gray. In L. M. Bloomingdale \& J. Sergeant (Eds.), Attention deficit disorder: Criteria, cognition, intervention (pp. 117-125): Oxford: Pergamon.

Quay, H. C. (1997). Inhibition and attention deficit hyperactivity disorder. Journal of Abnormal Child Psychology, 25, 7-13.

Rucklidge, J. J., \& Tannock, R. (2002). Neuropsychological profiles of adolescents with ADHD: Effects of reading difficulties and gender. Journal of Child Psychology and Psychiatry, 43, 988-1003.

Sattler, J. M. (2001). Assessment of children. Cognitive applications (4th ed.). San Diego: Jerome M. Sattler, Publisher, Inc.

Shader, R. I., Harmatz, J. S., Oesterheld, J. R., Parmelee, D. X., Sallee, F. R., \& Greenblatt, D. J. (1999). Population pharmacokinetics of methylphenidate in children with attention-deficit hyperactivity disorder. Journal of Clinical Pharmacology, 39, 775-785.

Shaw, G. A., \& Giambra, L. (1993). Task-unrelated thoughts of college students diagnosed as hyperactive in childhood. Developmental Neuropsychology, 9, 17-30.

Shue, K. L., \& Douglas, V. I. (1992). Attention deficit-hyperactivity disorder and the frontal lobe syndrome. Brain and Cognition, 20, 104-124.

Smith, B. H., Pelham, W. E., Gnagy, E., Brooke, M., \& Evans, S. (2000). The reliability, validity, and unique contributions of self-report by adolescents receiving treatment for attention-deficit/hyperactivity disorder. Journal of Consulting and Clinical Psychology, 68, 489499.

Slusarek, M., Velling, S., Bunk, D. \& Eggers, C. (2001). Motivational effects on inhibitory control in children with ADHD. Journal of the American Academy of Child and Adolescent Psychiatry, 40, $355-363$.

Trommer, B. L., Hoeppner, J. B., \& Lorber, R. \& Armstrong, K. L. (1988). The go-no-go paradigm in attention deficit disorder. Annals of Neurology, 24, 610-614.

Trommer, B. L., Hoeppner, J. B., \& Zecker, S. G. (1991). The go-no go test in attention deficit disorder is sensitive to methylphenidate. Journal of Child Neurology, 6(Suppl.), S126-S129.

Wallace, J., Bachorowski, J., \& Newman, J. P. (1991). Failures of response modulation: Impulsive behavior in anxious and impulsive individuals. Journal of Research in Personality, 25, 23-44.

Wallace, J., \& Newman, J. P. (1990). Differential effects of reward and punishment cues on response speed in anxious and impulsive individuals. Personality and Individual Differences, 11, 999-1009.

Wechsler, D. (1991). Manual for the WISC-III. New York, NY: Psychological Corporation.

Wechsler, D. (1997). Manual for the WISC-III. New York, NY: Psychological Corporation.

Wilkison, P. C., Kircher, J. C., McMahon, W. H., \& Sloane, H. N. (1995). Effects of methylphenidate on reward strength in boys with attention-deficit hyperactivity disorder. Journal of the American Academy of Child and Adolescent Psychiatry, 34, 897-901.

Zelazo, P. D., Reznick, J. S., \& Piñon, D. E. (1995). Response control and the execution of verbal rules. Developmental Psychology, 31, $508-517$. 